\title{
Context-Adaptive Recursive-Filtering-Based Intra Prediction in Video Coding
}

\author{
Hui Su \\ Google, USA \\ huisu@google.com \\ Debargha Mukherjee \\ Google, USA \\ debargha@google.com
}

\author{
Alexander Bokov \\ Google, USA \\ alexanderbokov@google.com \\ Jingning Han \\ Google, USA \\ jingning@google.com
}

\author{
Urvang Joshi \\ Google, USA \\ urvang@google.com \\ Yue Chen \\ Google, USA \\ yuec@google.com
}

\begin{abstract}
Conventional intra prediction modes in image and video coding generate an estimation of a target block by copying or projecting its causal neighboring pixels along certain angles. Such simple directional model does not work well for complex image structures. A set of context-adaptive intra prediction modes based on recursive filtering is proposed in this paper. The prediction of a block is generated by applying linear filtering over certain previously reconstructed or predicted pixels in the causal neighborhood of each pixel recursively. The filter coefficients are estimated with least squares optimization using previously reconstructed pixels in the above and/or left regions of the current block. The configurations for the filters such as filter taps, position of reference pixels, as well as the location and shape of the training regions are all flexible, making the proposed prediction modes highly adaptive to local image texture contexts. A data-driven approach is used to select the optimal subset of all the possible filter configurations while retaining as much coding gains as possible. The proposed approach is tested on the state-of-the-art AV1 video coding standard. AV1 supports sophisticated intra prediction tools such as recursive filtering, quadratic interpolation filtering, intra block-copy, and the palette mode. Experimental results show that the contextadaptive recursive-filtering-based intra prediction modes can achieve significant improvement in compression efficiency.
\end{abstract}

\section{CCS CONCEPTS}

- Information systems $\rightarrow$ Multimedia information systems.

\section{KEYWORDS}

Video Coding, Intra Prediction, Least Squares, Context Adaptive

Permission to make digital or hard copies of part or all of this work for personal or classroom use is granted without fee provided that copies are not made or distributed for profit or commercial advantage and that copies bear this notice and the full citation on the first page. Copyrights for third-party components of this work must be honored.

For all other uses, contact the owner/author(s).

PV'19, June 21, 2019, Amherst, MA USA

(C) 2019 Copyright held by the owner/author(s).

ACM ISBN 978-1-4503-6300-6/19/06.

https://doi.org/10.1145/3304114.3325615
ACM Reference Format:

Hui Su, Alexander Bokov, Urvang Joshi, Debargha Mukherjee, Jingning Han, and Yue Chen. 2019. Context-Adaptive RecursiveFiltering-Based Intra Prediction in Video Coding. In 24th Packet Video Workshop (PV'19), June 18, 2019, Amherst, MA, USA. ACM, New York, NY, USA, Article 1, 6 pages. https://doi.org/10. $1145 / 3304114.3325615$

\section{INTRODUCTION}

Intra prediction is a fundamental technique in block based image and video coding to reduce spatial redundancy between adjacent picture blocks. In recent generations of video compression standards[1, 12, 15, 16], conventional intra prediction modes typically generate estimates of the coding block by copying or projecting its previously reconstructed neighboring pixels along certain directions. This simple directional model assumes that all the pixels inside the coding block follow an approximately uniform gradient direction, and therefore cannot represent relatively more complex image textures and structures well. Linear filtering has been extensively explored to complement conventional directional intra prediction modes. In [8], a least squares based adaptive linear prediction mode is proposed for lossless compression of natural images. This approach is extended to video compression in [7] and [9], where the spatial and temporal neighbors of the current pixel or block are utilized for inter prediction. In [10], an adaptive linear filtering based approach is proposed as a new intra prediction mode. The filter coefficients are estimated with least squares optimization using previously reconstructed pixels in a training window. The training window can be either co-located in a previously reconstructed frame, or located near the current block in the same frame. The decoder can repeat the coefficient estimation process, therefore there is no need to signal any overhead for the coefficients in the bitstream. The authors in [17] model intra prediction as a linear combination of all the available reference pixels, and adapts the weight coefficients for each pixel based on its coordinates within the coding block. An extension to HEVC intra prediction that combines values predicted using nonfiltered and filtered reference samples is presented in [14]. Considering the image signal as a 2-D non-separable Markov model, Chen et al. proposes novel intra prediction modes that generate predictions with a set of predefined 3-tap recursive linear filters that are applied with the nearest causal pixel 
neighbors on top, left, and the top-left corner[2]. Pixels in a block are predicted recursively in raster scan order. The filter coefficients are obtained off-line using a "K-modes" iterative approach to minimize the overall rate-distortion cost. The choice of which set of filter coefficients to use for each coding block is encoded explicitly in the bitstream. In [6], the authors extend the work to 4 -tap recursive filters utilizing either the pixel at the top-right or bottom-left corner as well. The recursive-filtering-based intra prediction approach has been considered and experimented by the state-of-the-art open source video codec format AV1[5]. The final version of the recursive-filtering-based intra prediction modes adopted by AV1, namely the "FILTER_INTRA" modes, is optimized for tradeoff between compression efficiency and feasibility for SIMD implementation[3]. The FILTER_INTRA modes predict a block on the basis of $4 \times 2$ pixel patches. Each $4 \times 2$ patch is generated with linear filtering over its 7 causal pixel neighbors, and there are in total 5 different predefined sets of filter coefficients.

In this paper, we propose a set of context-adaptive intra prediction modes based on recursive filtering, namely the "CARF" (context-adaptive recursive-filtering) modes. The prediction of a block is generated by applying linear filtering over certain previously reconstructed/predicted pixel values in the causal neighborhood of each pixel in the block recursively, in the row-major or column-major order. The filter coefficients are obtained with least squares optimization using previously reconstructed pixels in the above and/or left regions of the current block. What makes the CARF modes superior to previous work is that, to be highly adaptive to local image texture characteristics, the configurations of the filters, such as the number of filter taps, position of reference pixels, as well as the location and shape of the training regions are all flexible. Given the freedom of adjusting these configurations, lots of new CARF prediction modes can be devised to complement conventional intra prediction in an image/video codec. To reduce the complexity for the encoder to select the best mode for each block in rate-distortion optimization, and the bitstream overhead to signal the selected mode, we use a data-driven approach to identify only a small subset of all the possible CARF modes to achieve the majority of the compression improvement. The proposed approach can work with any block-based codec. We have conducted experiments with the newest video compression format AV1, which supports quite a few sophisticated intra prediction tools such as recursive filtering, quadratic interpolation filtering, intra block-copy, and the palette mode. The experimental results show that the CARF modes can achieve significant improvement in compression efficiency for AV1.

The remainder of the paper is organized as follows: Section 2 presents the proposed CARF modes in details. In Section 3 , we introduce a data-driven method to identify the optimal subset of the CARF modes that can retain the majority of the compression gains. Some experimental results on AV1 are presented in Section 4, and finally Section 5 concludes the paper.

\section{CONTEXT-ADAPTIVE RECURSIVE-FILTERING-BASED INTRA MODES}

The proposed context-adaptive recursive-filtering(CARF)based intra modes generate the prediction of each pixel in a coding block by recursively applying linear filtering on a set of adjacent reference pixels that are previously reconstructed/predicted. The prediction of a pixel will be used as a reference value for later pixels. The filter coefficients are derived on the fly for each coding block, so that they are adaptive to the local image texture context of the coding block. To perform such online training, we consider the following least squares optimization problem:

$$
\begin{aligned}
\left(\alpha_{1}, \alpha_{2}, \ldots \alpha_{n}\right)=\arg \min _{\alpha 1, \ldots, \alpha_{n}}[ & \sum_{x \in R}\left(x-\sum_{i=1, \ldots n}\left(\alpha_{i} \cdot x_{i}\right)\right)^{2} \\
& \left.+\lambda\left(\alpha_{1}^{2}+\ldots+\alpha_{n}^{2}\right)\right]
\end{aligned}
$$

Here, $R$ denotes the training region that contains the set of pixels for which we fit the optimal $n$-tap filter coefficients $\left(\alpha_{1}, \ldots, \alpha_{n}\right)$ in the least squares sense. $\left(x_{1}, \ldots, x_{n}\right)$ are the sets of reference pixels that are used for predicting pixel $x$ in the training region. The pixels in the training region $R$ are previously reconstructed and are available for both the encoder and decoder. Therefore we can simply repeat the same computation at the decoder end, instead of explicitly signalling the coefficients in the bitstream. $\lambda$ denotes the regularization term that favors solutions that are closer to zero, all else being equal. Such regularization guarantees that the problem has a unique solution. For example, with $\lambda=0$ and $x=$ aconstant value for all $x \in R$, there are infinitely many solutions if there is no regularization.

As illustrated in Fig. 1, there are 5 different reference pixel positions under consideration: on the top, left, top-left, top-right, and bottom-left. Note that the bottom-left and topright pixels cannot be used at the same time, because using top-right pixel requires the row-major prediction order, while using bottom-left pixel requires the column-major prediction order. With this constraint, the possible number of filter taps can be 2,3 and 4 .

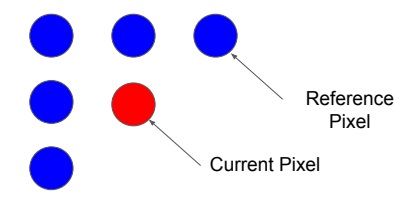

Figure 1: Reference pixels used for filtering.

We consider all the possible 2-tap, 3-tap and 4-tap filters that use a certain subset of the 5 possible reference pixels, with the limitation that the top-right and bottom-left pixels 


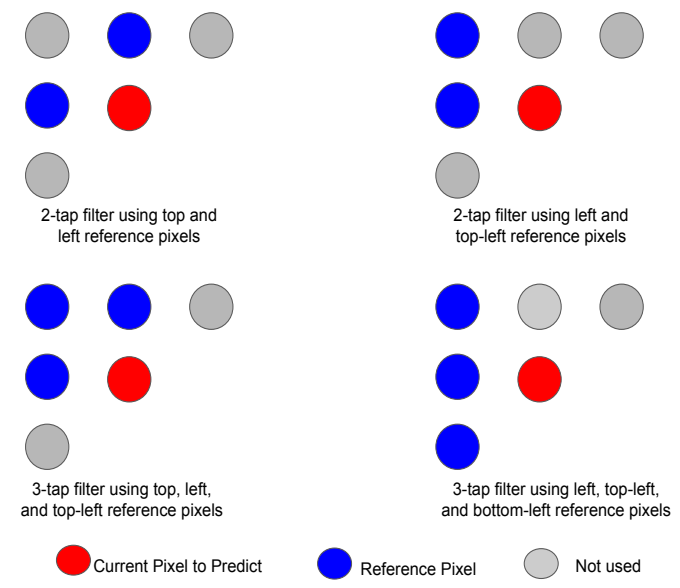

Figure 2: Different filter tap number and reference pixel position configurations.

cannot be used at the same time. This leads to 18 possible choices for filter tap number and position combinations. Fig. 2 shows some examples of 2-tap and 3-tap filter configurations.

For additional flexibility we also consider fitting each filter on 3 possible training regions: full context, only left context, and only top context, as shown in Fig 3 . The flexibility to use different training regions is beneficial because it provides robustness against distortions that is inevitable for lossy compression. The reconstructed pixel values are used for estimating the filter coefficients. If the pixels in a certain region suffer severe quantization distortions, then fitting the filter coefficients over that region may result in poor results. So it is possible to improve prediction accuracy by using only a partial context. In total, we have $3 \times 18=54$ possible combinations of filter tap number, reference pixel position, and training region configurations.

\section{SELECTING THE MOST EFFECTIVE FILTERS}

It is not ideal to support all of the $54 \mathrm{CARF}$ intra prediction modes(each corresponding to a unique filter configuration) in a practical video codec. Excessive number of modes requires more overhead in the bitstream to signal the selected mode for each coding block. It increases complexity for the implementation of the encoder and the decoder as they both need support all the prediction modes. It also poses significant computational challenge for an encoder if the mode decisions are obtained with exhaustive rate-distortion search among all the possible prediction mode candidates. Therefore, it is desired to utilize only a small subset of all the CARF modes while still being able to achieve as much compression gains as possible.

It is difficult to determine which subset of the CARF modes should be the most effective ones with either theoretical or empirical analysis. We propose a data-driven approach to

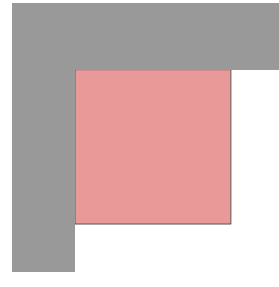

Full Context

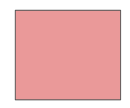

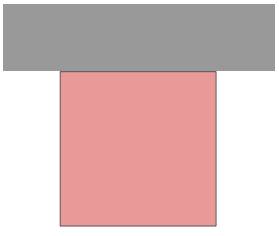

Top Context

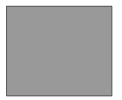

Training Region
Figure 3: Different training regions for filter coefficient fitting.

tackle this problem. Specifically, we consider the following minimization problem:

$$
\begin{array}{r}
S_{k}=\arg \min _{S \in P_{k}(F)}\left[\sum _ { b \in B } \operatorname { m i n } \left(M S E_{\text {best_mode }}(b),\right.\right. \\
\left.\left.\min _{f \in S}\left(M S E_{f}(b)\right)\right)\right]
\end{array}
$$

Here, $F$ is the full set of 54 filters; $P_{k}(F)$ is the set of all size- $k$ subsets of $F$; $B$ is a sufficiently large training set of pixel blocks from pictures of varying contents and resolutions; $M S E_{\text {best_mode }}(b)$ is the mean square error(MSE) that we can obtain when predicting the block $b$ using the best out of the existing intra prediction modes; $M S E_{f}(b)$ denotes the MSE that we can achieve when using a particular contextadaptive filter $f$. The solution to this optimization problem is essentially the top $k \mathrm{CARF}$ modes for the purpose of minimizing the average MSE of predicting blocks in the training set.

Experiments are conducted on AV1, and the results for different values of $k$ are shown in Fig. 4 . We see that the average MSE(for intra prediction) is reduced by about $23 \%$ if all the $54 \mathrm{CARF}$ intra modes are added to AV1. It is interesting to observe that the top 10 filters, which can provide about $15 \%$ average MSE reduction, are all 2-tap filters. A possible explanation is that using more filter taps can cause the overfitting problem. The filter coefficients are estimated using pixels adjacent to, but outside, the coding block. Also, the pixel values are reconstructed values as opposed to original source values. The pixels in the coding block may therefore have different texture statistics from the pixels in the training regions. More sophisticated filters with higher filter taps may fit the training pixel values better, but do not necessarily predict the coding block better. We can also see that the flexibility to choose training regions among full, top and left contexts is beneficial. Some of the top effective filters are estimated with a partial context(either top or left) instead of 
the full context. Another interesting observation is that the most effective filters demonstrate some symmetric structures. For example, the $2 n d$ and $3 r d$ filters use pixels on transposing positions and alternate training regions. This is the same for the $4 t h$ and $5 t h$ filters, the $6 t h$ and $7 t h$ filters, etc. This verifies that the data-driven approach can indeed capture which filters are the most effective, rather than randomly picking some filters as the top candidates.

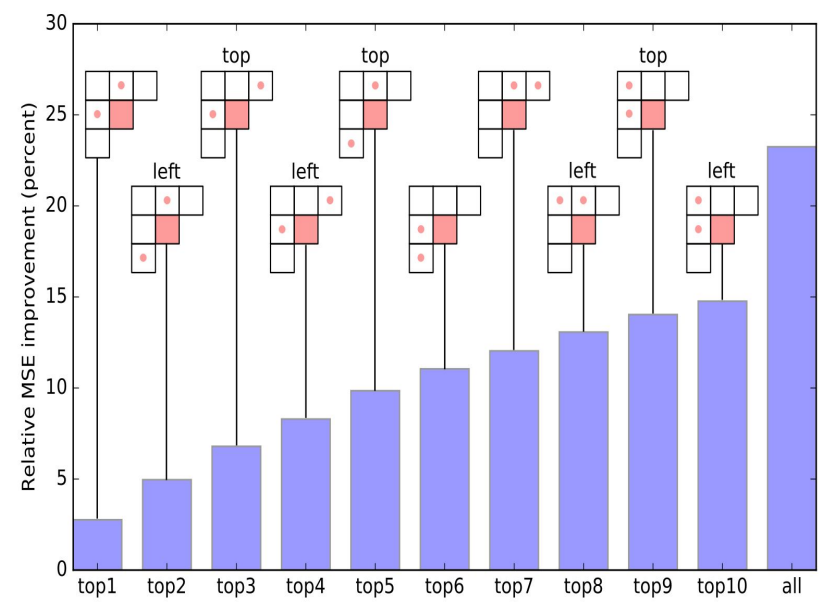

Figure 4: Best $k$ adaptive filter configurations for the purpose of minimizing prediction MSE. Red dots denote the filter tap positions. If the filter uses either only top or only left context for filter coefficient fitting, it's marked accordingly.

We realize that the filters that are the most effective at reducing MSE may not necessarily be the best ones in terms of improving the overall compression performance, which is our ultimate goal. So instead of optimizing for minimized MSE, it is more sensible to optimize for minimized ratedistortion(RD) cost. With the new optimization goal, Eq. 2 becomes Eq. 3. Fig. 5 shows the results obtained on AV1. We can see that the RD cost reduction is about $5.5 \%$ when all the 54 CARF modes are utilized. The best 10 filters, most of which have 3 or 4 taps, are quite different from the filters that we obtain when optimizing for minimized MSE. The top 10 filters can provide about $3 \% \mathrm{RD}$ cost reduction. It should be noted that at this point we have not accounted for the extra bitstream overhead for signaling the choice of CARF mode at prediction unit level. So the actual compression improvement percentage should be less than the $\mathrm{RD}$ cost reduction.

$$
\begin{array}{r}
S_{k}=\arg \min _{S \in P_{k}(F)}\left[\sum _ { b \in B } \operatorname { m i n } \left(R D_{\text {best_mode }}(b),\right.\right. \\
\left.\left.\min _{f \in S}\left(R D_{f}(b)\right)\right)\right]
\end{array}
$$

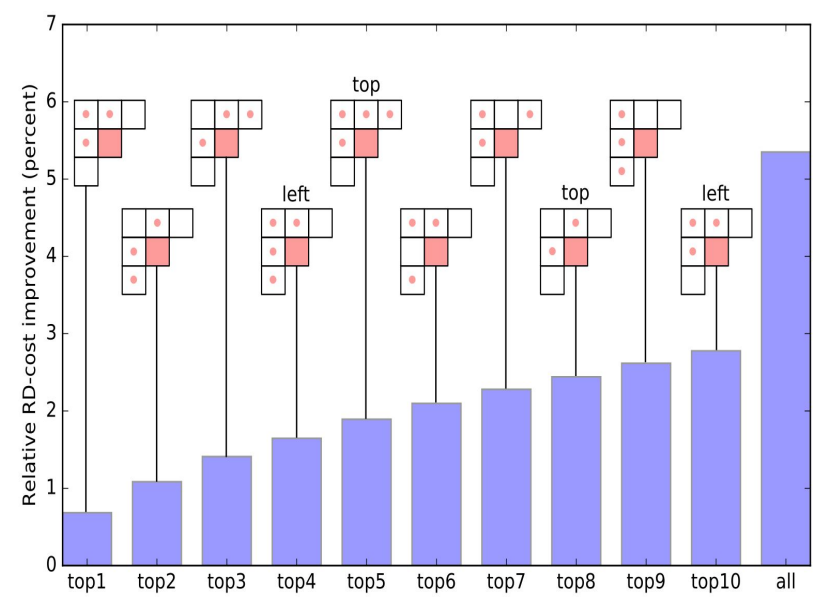

Figure 5: Best filter configurations for the purpose of minimizing RD cost. Red dots denote the filter tap positions. If the filter uses either only top or only left context for filter coefficient fitting, it's marked accordingly.

So far, we have been assuming that the shape and size of the training regions that contain the set of pixels for which we fit the filter coefficients are fixed as 4 rows on top and/or 4 columns to the left. If we optimize the shape and size of the training regions, we may obtain additional compression improvement. To find the optimal shape and size of the training regions, we introduce the parameterization $w=\left(w_{1}, w_{2}, w_{3}, w_{4}\right)$, as illustrated in Fig 6 . Some pixel values may not be available in certain non-causal areas within the training regions, e.g. on the top-right and bottom-left of the current block. In that case, we simply discard the pixels in the non-causal areas. To find the best shape parameters, we solve the following optimization problem separately for each prediction unit size:

$$
\begin{aligned}
& \left(w^{1}, \ldots, w^{k}\right)= \\
& \arg \min _{w^{1}, \ldots, w^{k}}\left[\sum _ { b \in B } \operatorname { m i n } \left(R D_{\text {best_mode }}(b),\right.\right. \\
& \left.\left.\min _{i \in[1, k]}\left(R D_{\text {adapt }}^{i}\left(w^{i}, b\right)\right)\right)\right]
\end{aligned}
$$

Here, $\left(w^{1}, \ldots, w^{k}\right)$ are the shape parameters of the training regions for each of $k$ previously selected CARF modes; $B$ denotes the set of training blocks of a certain size; $R D_{\text {adapt }}^{i}\left(w^{i}, b\right)$ is the $\mathrm{RD}$ cost of using the $i-t h$ adaptive CARF mode with $w^{i}$ as the training region shape parameters for the coding block $b$.

A brute-force approach of trying every possible combination of $\left(w^{1}, \ldots, w^{k}\right)$ is prohibitively computationally expensive, so we resort to a greedy approximate minimization algorithm. 


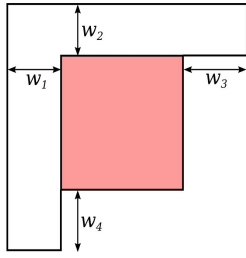

(a) Full context

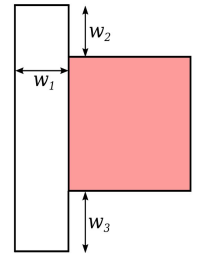

(b) Left context

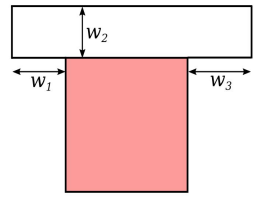

(c) Top context
Figure 6: Shape parameterization for three types of training regions used for CARF. All previously reconstructed pixels within these regions are used for fitting the filter coefficients, which is then used for predicting the current coding block marked in red.

Starting with some reasonable initial approximation, we iteratively pick a mode that provides the best compression improvement, and modify its training region shape parameters while keeping all the other modes unchanged. To further narrow down the search space, we only consider the training region shape modifications in a small neighborhood around the current one. This iterative process is repeated until convergence.

\section{EXPERIMENTAL RESULTS}

In this section, we demonstrate the effectiveness of the proposed CARF based intra prediction modes with experimental results obtained on AV1. AV1 is a state-of-the-art open-source and royalty-free video compression format that is jointly developed and finalized in early 2018 by the Alliance for Open Media(AOM) industry consortium[4]. It can achieve significant bitrate savings over previous codecs such as VP9 and HEVC, thanks to numerous sophisticated coding tools[3]. Specifically for intra prediction, AV1 supports up to 56 directional modes, quadratic interpolation filtering modes, the palette mode, the intra block-copy mode, as well as a SIMD friendly variation of the recursive-filtering-based modes introduced in $[2,6]$.

In our experiment, the proposed CARF based predictors are implemented as additional intra modes in the reference software codec of AV1[13]. The new intra modes can be chosen for the luma and chroma components seperately. We vary the number of the CARF modes that are added. The filter tap number, reference pixel position and training region configurations are selected according to the method described in Sec. 3, after which the training region shape parameters are optimized. Compression performance is compared between AV1 encoder with and without CARF modes. Two test sets covering different video resolutions are considered. The medium resolution testset contains about $30480 \mathrm{P}$ video clips; the high resolution testset contains about $40720 \mathrm{P}$ and $1080 \mathrm{P}$ video clips. The test sequences are typical test materials, and many of them are from the "derf" test set [11]. As
Table 1: Average coding gains of the CARF modes for AV1 intra coding

\begin{tabular}{ccc}
\hline $\begin{array}{c}\text { Number of CARF } \\
\text { modes added }\end{array}$ & $\begin{array}{c}\text { Medium resolution } \\
(480 \mathrm{P})\end{array}$ & $\begin{array}{c}\text { High resolution } \\
(720 \mathrm{P} \text { and 1080P })\end{array}$ \\
\hline 3 & $0.72 \%$ & $0.85 \%$ \\
5 & $0.95 \%$ & $1.13 \%$ \\
7 & $1.09 \%$ & $1.21 \%$ \\
7 with Fixed & $0.79 \%$ & $1.03 \%$ \\
Training Regions & & \\
\hline
\end{tabular}

Table 2: Notable coding gains with $7 \mathrm{CARF}$ modes added to AV1

\begin{tabular}{cc}
\hline Test Sequence & Coding Gains \\
\hline dinner_1080P & $6.82 \%$ \\
sintel_trailer_480P & $6.72 \%$ \\
sunflower_720P & $2.63 \%$ \\
pedestrian_area_1080P & $2.45 \%$ \\
rush_hour_1080P & $2.30 \%$ \\
BasketballDrillText_832x480 & $2.09 \%$ \\
Flowervase_832x480 & $1.92 \%$ \\
tennis_1080p & $1.84 \%$ \\
\hline
\end{tabular}

the proposed method only affects intra prediction efficiency, we encode every frame as a key-frame. Each sequence is encoded with various quantization parameters to cover different bitrate settings.

The experimental results are shown in Table 1. Averaging across all the test sequences, up to $1.2 \%$ BD-rate reduction is observed when adding 7 CARF modes to AV1; $0.9 \% \sim 1.1 \%$ $\mathrm{BD}$-rate reduction when adding 5 CARF modes; $0.7 \% \sim 0.8 \%$ BD-rate reduction when adding only $3 \mathrm{CARF}$ modes. The compression improvement is consistent between the 2 test sets, while slightly larger gains are observed for videos of higher resolutions. Some notable compression gains(with 7 CARF modes added) are listed in Table 2. The largest BDrate reduction is as much as $6.8 \%$. We also observe that the optimization of the shape and size of the training regions gives us decent additional compression gains. If the training region shape parameters are fixed as $w_{1}=w_{2}=w_{3}=w_{4}$ $=4$, the compression gains are reduced by as much as more than $20 \%$.

We also conducted experiments to compare the proposed CARF modes with AV1's existing recursive-filtering-based intra modes, namely the "FILTER_INTRA" modes. AV1's FILTER_INTRA modes predict the $4 \times 2$ pixel patches of a block recursively, and each patch is generated with linear filtering over its 7 causal pixel neighbors. There are 5 different predefined sets of filter coefficients available. We use the AV1 encoder with all the FILTER_INTRA modes disabled as the baseline and repeat the coding efficiency comparison 
Table 3: CARF modes vs. FILTER_INTRA modes in AV1

\begin{tabular}{ccc}
\hline & $\begin{array}{c}\text { Medium resolution } \\
(480 \mathrm{P})\end{array}$ & $\begin{array}{c}\text { High resolution } \\
(720 \mathrm{P} \text { and 1080P) }\end{array}$ \\
\hline FILTER_INTRA & $0.63 \%$ & $0.64 \%$ \\
CARF & $1.59 \%$ & $1.67 \%$ \\
Both & $1.72 \%$ & $1.83 \%$ \\
\hline
\end{tabular}

Table 4: Decoder complexity analysis

\begin{tabular}{cc}
\hline & Decoding Time Increase \\
\hline Average & $6.5 \%$ \\
Max & $9.8 \%$ \\
Min & $2.8 \%$ \\
\hline
\end{tabular}

experiment. Table 3 shows the results with 7 CARF modes and 5 FILTER_INTRA modes. We can see that the CARF modes achieve about $1.6 \%$ compression improvement for AV1 with FILTER_INTRA modes disabled. It is much larger compression improvement than the FILTER_INTRA modes. The FILTER_INTRA modes only provide very marginal improvement on top of the CARF modes.

The CARF modes require filter coefficient estimation on both the encoder and decoder sides. Decoder computational complexity may become a bottleneck for some video compression applications. We have conducted experiments to test the impact of the CARF modes on decoding speed. Test sequences are encoded with $7 \mathrm{CARF}$ modes enabled. The decoding time is compared with the AV1 baseline. As shown by the results listed in Table 4 , the decoding time increase is in the range of $2.8 \%$ to $9.8 \%, 6.5 \%$ on average. It should be noted that our current implementation is in plain $\mathrm{C}$ with double precision. Lots of potential optimization work can be done to reduce the computational complexity overhead, such as integerization and SIMD implementations. It is also possible to adjust the scheme to obtain appropriate trade-off between compression performance and computational complexity. For example, one can abandon the 4-tap filters and only consider 2 -tap and 3 -tap ones.

\section{CONCLUSIONS}

A set of novel intra prediction modes based on contextadaptive recursive-filtering $(\mathrm{CARF})$ are proposed. The prediction of a block is generated by applying linear filtering over the previously reconstructed/predicted pixel values in the causal neighborhood of each pixel recursively. The filter coefficients are estimated with least squares optimization using previously reconstructed pixels in the above and/or left regions of the coding block. To be adaptive to local image texture characteristics, the number of filter taps, the position of reference pixels used for filtering, as well as the location and shape of the filter coefficient fitting regions are all flexible. A data-driven approach is used to select a small subset of all the possible CARF modes while retaining the majority of the compression gains. Experiments conducted with the AV1 video codec show that the proposed CARF modes can achieve significant compression gains for AV1 intra frame coding, in spite of AV1's existing sophisticated intra prediction modes.

\section{REFERENCES}

[1] Jim Bankoski, Paul Wilkins, and Yaowu Xu. 2011. Technical overview of VP8, an open source video codec for the web. In IEEE International Conference on Multimedia and Expo.

[2] Y. Chen, J. Han, and K. Rose. 2013. A Recursive Extrapolation Approach to Intra Prediction in Video Coding. In Proc. IEEE International Conference on Acoustics, Speech, and Signal Processing (ICASSP).

[3] Y. Chen et al. 2018. An Overview of Core Coding Tools in the AV1 Video Codec. In Picture Coding Symposium.

[4] Alliance for Open Media. [n. d.]. https://aomedia.org/

[5] U. Joshi, D. Mukherjee, J. Han, Y. Chen, S. Parker, H. Su, A. Chiang, Y. Xu, Z. Liu, Y. Wang, J. Bankoski, C. Wang, , and E. Keyder. 2017. Novel inter and intra prediction tools under consideration for the emerging AV1 video codec. In Proc. SPIE, Applications of Digital Image Processing XL.

[6] S. Li, Y. Chen, J. Han, T. Nanjundaswamy, and K. Rose. 2016. Rate-Distortion Optimization and Adaptation of Intra Prediction Filter Parameters. In Proc. IEEE International Conference on Image Processing (ICIP).

[7] X. Li. 2006. Least-Square Prediction for Backward Adaptive Video Coding. EURASIP Journal on Advances in Signal Processing (2006).

[8] X. Li and M.T. Orchard. 2001. Edge-directed prediction for lossless compression of natural images. IEEE Transactions on Image Processing 10, 6 (June 2001), 813-817.

[9] L. Liu, Y. Liu, and E. J. Delp. 2007. Content-adaptive motion estimation for efficient video compression. In Proceedings of the SPIE International Conference on Visual Communications and Image Processing.

[10] Limin Liu, Yuxin (Zoe) Liu, and Edward J. Delp. 2007. Enhanced intra prediction using context-adaptive linear prediction. In Picture Coding Symposium.

[11] Xiph.org Video Test Media. [n. d.]. https://media.xiph.org/ video/derf/

[12] Debargha Mukherjee, Jingning Han, Jim Bankoski, Ronald Bultje, Adrian Grange, John Koleszar, Paul Wilkins, and Yaowu Xu. 2015. A Technical Overview of VP9-The Latest Open-Source Video Codec. Proc. SMPTE Motion Imaging Journal 124, 1 (Jan. 2015).

[13] AV1 reference software codec. [n. d.]. https://aomedia. googlesource.com/

[14] A. Said, X. Zhao, M. Karczewicz, J. Chen, and F. Zou. 2016. Position dependent prediction combination for intra-frame video coding. In Proc. IEEE International Conference on Image Processing (ICIP).

[15] G. J. Sullivan, J.-R. Ohm, W.-J. Han, and T. Wiegand. 2012. Overview of the High Efficiency Video Coding (HEVC) standard. IEEE Trans. Circuits Syst. Video Technol. 22, 12 (Dec. 2012), 1648-1667.

[16] T. Wiegand, G.J. Sullivan, G. Bjontegaard, and A. Luthra. 2003. Overview of the H.264/AVC Video Coding Standard. IEEE Trans. Circuits Syst. Video Technol. 13, 7 (July 2003).

[17] L. Zhang, X. Zhao, S. Ma, Q. Wang, and W. Gao. 2011. Novel intra prediction via position-dependent filtering. Journal of Visual Communication and Image Representation 22 (Nov. 2011), 687696. 